\title{
Scanning Fourier transform spectrometer in the visible range based on birefringent wedges
}

\author{
Aurelio Oriana ${ }^{1}$, Julien Réhault ${ }^{2}$, Fabrizio Preda ${ }^{1}$, Dario Polli ${ }^{1}$, And \\ Giulio Cerullo1,* \\ ${ }^{1}$ IFN, Dipartimento di Fisica, Politecnico di Milano, Piazza Leonardo da Vinci 32, I-20133 Milano, Italy \\ 2 Paul Scherrer Institute, SwissFEL, 5232 Villigen, Switzerland \\ *Corresponding author: giulio.cerullo@polimi.it
}

Received 16 March 2016; revised 1 June 2016; accepted 9 June 2016; posted 10 June 2016 (Doc. ID 260957); published 27 June 2016

\begin{abstract}
We introduce a spectrometer capable of measuring sample absorption spectra in the visible, based on a time-domain scanning Fourier-transform (FT) approach. While infrared FT spectrometers (FTIR) typically employ a Michelson interferometer to create the two delayed light replicas, the proposed apparatus exploits a compact common-mode passive interferometer that relies on the use of birefringent wedges. This ensures excellent path-length stability $(\sim \lambda / 300)$ and accuracy, with no need for active feedback or beam tracking. We demonstrate the robustness of the technique measuring the transmission spectrum of a colored bandpass filter over one octave of bandwidth and comparing the results with those obtained with a commercial spectrophotometer.
\end{abstract}

(C) 2016 Optical Society of America One print or electronic copy may be made for personal use only. Systematic reproduction and distribution, duplication of any material in this paper for a fee or for commercial purposes, or modifications of the content of this paper are prohibited.

OCIS codes: (070.4790) Spectrum analysis; (120.3180) Interferometry; (260.1440) Birefringence; (300.6300) Spectroscopy, Fourier transforms.

http://dx.doi.org/10.1364/JOSAA.33.001415

\section{INTRODUCTION}

Optical spectrometers can be broadly divided in frequency-domain and time-domain ones. Frequency-domain spectrometers rely on a dispersive element, such as a grating or a prism, to spatially separate the different frequency components of the light field, which are either serially scanned on a single detector or measured in parallel by a multichannel detector, such as a photodiode array or a CCD. In the timedomain, Fourier-transform (FT) spectrometers [1, 2] use an interferometer, which creates two collinear replicas of the input light radiation $E(t)$ and $E(t-\tau)$, where $\tau$ is their relative delay. The interference of these replicas, recorded by a single detector as a function of $\tau$, gives rise to an interferogram:

$$
\begin{aligned}
\mathrm{I}(\tau) & =\int|\mathrm{E}(\mathrm{t})+\mathrm{E}(\mathrm{t}-\tau)|^{2} d t \\
& =2 \int|\mathrm{E}(\mathrm{t})|^{2} d t+\int \mathrm{E}(\mathrm{t}) \mathrm{E}^{*}(\mathrm{t}-\tau) d t+c c
\end{aligned}
$$

The first term of Eq. (1) is a DC offset that does not depend on $\tau$. By subtracting it, we are left with the rapidly oscillating AC component that brings information on the spectrum of $E(t)$ :

$$
\mathrm{I}_{A C}(\tau)=\int \mathrm{E}(\mathrm{t}) \mathrm{E}^{*}(\mathrm{t}-\tau) d t+c c
$$

By computing the FT of Eq. (2) we obtain after some simple steps:

$$
\tilde{\mathrm{I}}_{A C}(\omega)==\widetilde{\mathrm{E}}^{*}(\omega) \cdot \widetilde{\mathrm{E}}(\omega)+c c=2|\widetilde{\mathrm{E}}(\omega)|^{2}=2 \tilde{\mathrm{I}}(\omega)
$$

This equation (also known as Wiener-Khintchine theorem) demonstrates that the FT of the (AC component of the) interferogram directly provides the spectrum $I(\omega)$ of the incoming light.

FT spectrometers have found a great number of applications in astronomy [3], molecular sciences [4, 5], biology [6, 7], atmospheric sciences [8] and many more. They are widely employed in the midinfrared spectral range (FTIR) [1], where multiplex detectors are expensive and not readily available, and in nuclear magnetic resonance [4], but their principle can be applied to any part of the electromagnetic spectrum. In the mid-infrared, the sensitivity is limited by the detector noise [9] and the use of a single detector, as in FTIR spectrometers, provides an improvement with respect to a detector array (the so-called Felgett's advantage [10]). In the visible range, on the other hand, lowcost multiplex detectors are widespread and turn out to be advantageous since sensitivity is limited by shot noise rather than detector noise [11]. Nevertheless, time-domain spectrometers retain the advantages of (i) high throughput, due to the lack of slits (the Jacquinot advantage) $[12,13]$; (ii) high absolute frequency accuracy, thanks to the possibility of calibrating the interferometer with a known light source (the Connes advantage); (iii) high frequency resolution, limited only by the scanning range of the interferometer, and (iv) broad wavelength coverage, as determined by the spectral responsivity of the 
detector. These four advantages motivate the extension of FT spectrometers to the visible and UV ranges.

Typically, FT spectrometers use a Michelson interferometer (MI) to create the two delayed beam replicas (Fig. 1(a)) [14, 15]. In a MI the incoming light beam is divided by a beam splitter into two replicas of the same amplitude, which are reflected back by two mirrors, one stationary and the other moving, and cross again the beam splitter where they are recombined. The replicas interfere at the detector so that the measured light intensity depends on the path-length difference of the two arms of the interferometer, set by the instantaneous position of the moving mirror. An FT spectrometer requires control of the pathlength difference of the interferometer arms with accuracy much better than the optical wavelength, which is typically obtained by active stabilization or by tracking with an auxiliary beam. For the relatively long mid-infrared wavelengths this is easily achieved by an auxiliary visible beam, such as a He-Ne laser; when moving to shorter visible/UV wavelengths, the demands on positioning accuracy of the interferometer become increasingly difficult to satisfy, calling for complicated electronic, optical and mechanical setups. Cageao [16] used a heterodyne interferometer, in which a phase-locked loop locks the phase difference between a reference beat frequency signal and a measurement beat frequency signal to feedback control the speed of the moving mirror. In the so-called beam-folding technique, introduced by Chan $[17,18]$, a multipath auxiliary interferometer is used to increase the positioning accuracy of the tracking beam. A two-output steppingmode interferometer has been proposed by Mandon [19], where laser frequency combs are used as light sources for FT spectroscopy, increasing sensitivity by several orders of magnitude: here, two fast InGaAs detectors measure the interferogram as a function of the optical path difference between the interferometer arms. Alternatively, static FT spectrometers have been demonstrated [20, 21], in which a Wollaston prism (WP) between crossed polarizers introduces a delay between orthogonal polarizations which varies along the transverse coordinate: by imaging such transverse profile on a CCD camera, one obtains interference fringes in the spatial rather than in the temporal domain, resulting in an inherently stable single-shot interferogram with no moving parts. A further implementation concerning a multichannel FT spectrometer which employs a WP is reported in [22]. Birefringence is also used in FT imaging spectrometers, which offer significant advantages over implementations using MIs [23-29]. Birefringent interferometers retain the advantages of traditional FT instruments but are inherently insensitive to mechanical vibrations thanks to their compact and common-path architecture. In the '60s Mertz illustrated a birefringent polarization version of a MI, developed for astronomy applications [23]. The light from a star, collected through a telescope, was sent to a WP generating two diverging beams with perpendicular polarization. The beams traversed a motorized Soleil compensator oriented at $45^{\circ}$ and two crossed polarizers and were then simultaneously detected using a photomultiplier. In this way it has been possible to measure spectra of stars and nebulae with the advantages of higher throughput and relaxed requirements in pointing accuracy with respect to standard spectrometers using slits. On the basis of the Mertz instrument, A'Hearn et al. [24] developed another polarization FT spectrometer based on a Babinet-Soleil compensator, which enabled them to record symmetric interferograms (i.e. also accessing the region around zero optical path difference) and to avoid chromatic dispersion of the light crossing a single prism. In the hyperspectral imaging technique introduced by Harvey [25], a scanning birefringent interferometer based on two WPs is used to create interference between two equal-amplitude, orthogonally polarized components of the input light, which are imaged on a CCD camera. The apparatus is in fact a polarizing interferometer, since it introduces a path difference between the orthogonally polarized components. This path difference is uniform across the width of the WPs, and is modulated by translation of one of the two prisms.

In this paper we present a compact scanning FT spectrometer working in the visible spectral range with inherently high path-length stability, without the need of active feedback or beam tracking. Our approach exploits a passive birefringent interferometer, recently introduced by our group and called Translating-Wedge-based Identical pulses eNcoding System (TWINS) [30-32], here proposed in a remarkably simplified version, to create two phase-locked replicas of a light beam to be measured and control their delay with ultrahigh accuracy, thanks to its common-mode architecture. We demonstrate the robustness of the technique by precisely measuring the transmission spectrum of a bandpass filter in the visible.

\section{PRINCIPLE OF OPERATION}

In TWINS the energy of the input light beam is equally distributed into two perpendicularly polarized components that collinearly propagate along the fast and slow axes of the birefringent material. The delay between these two components can be precisely controlled by varying the thickness of the birefringent material, which is shaped in a sequence of wedges. The TWINS system, originally developed for twodimensional spectroscopy and described in detail in [30-33], is inspired by the Babinet-Soleil compensator. While the latter is typically used as a variable waveplate, with total retardation of one wave or a few, the TWINS system is conceived so as to provide retardation of hundreds of optical cycles. It is here employed in a simplified version, lacking the two isotropic wedges (with optical axis aligned along the propagation direction). In our application, in fact, the absolute arrival time of one of the two replicas does not need to be kept constant in an absolute temporal reference frame (a fundamental requirement in two-dimensional spectroscopy) and the dispersion introduced by the varying thickness of the wedges does not constitute a problem, since we perform linear measurements. The setup is schematized in Fig. 1(b)-(c): it consists of two blocks ( $A$ and $B$ ), made of the same birefringent material and with optical axes aligned as shown in Fig. 1(c) (red arrows). Block A is a plate with fixed thickness $d_{A}$ and optical axis aligned along the y direction; block B consists of two wedges with an overall variable thickness $d_{B}$ and the optical axis aligned along the $x$ direction. After crossing blocks A and B, the two perpendicular polarizations are delayed by the quantity:

$$
\tau=\left(d_{A}-d_{B}\right)\left(\frac{1}{v_{g o}}-\frac{1}{v_{g e}}\right)
$$

where $v_{g o}$ and $v_{g e}$ are the ordinary and extraordinary group velocities, respectively. Fine tuning of the insertion of one of the two wedges of block $B$ allows varying the delay $\tau$. Note that the direction of motion is not exactly perpendicular to the light beam but inclined by the apex angle of the wedge (see orange arrow in Fig. 1(b)): in this way, the distance between the two wedges is kept constant, to minimize the lateral displacement of the beam during motion and thus maximize the fringe contrast in the recorded interferograms. The role of block $A$ is the same as the beam splitter of a MI, since it creates two replicas of the input beam, while one of the wedges of block B acts like the movable mirror of the MI, since it scans the delay between the two beams. Finally, the polarizer $\mathrm{P}$ before the photodetector acts like the second reflection onto the beam splitter of the MI, projecting the replicas onto the common polarization direction and thus allowing one to record their interferogram. For the sake of precision, TWINS is more similar to a Mach-Zehnder interferometer because each of the separated light paths is traversed only once in a single direction and then recombined in a different position (i.e. not using the same beam splitter as the one 


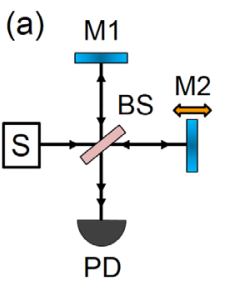

(b)


Fig. 1. Conceptual scheme of a Michelson interferometer (a) and a simplified version of TWINS interferometer (b). Double-sided orange arrows indicate the moving optics. S: light source; BS: beam splitter; M1: fixed mirror; M2: scanning mirror; PD: photodetector; P: polarizer. (c) Three-dimensional view of the TWINS: double-side red arrow indicates the optical axis of the birefringent material; $d_{A}$ and $d_{B}$ are the fixed and variable thicknesses of blocks A and B, respectively. Orange arrow indicates the direction of translation of a single wedge of block B..

for their separation). TWINS has the following advantages: (i) since both replicas follow the same optical path, their relative delay is locked with very high stability and reproducibility and is completely insensitive to mechanical fluctuations, so that no feedback stabilization or position tracking is required, in contrast to standard MIs; (ii) due to the small apex angle of the wedges and the typically small difference between $v_{g o}$ and vge, the device demultiplies the transverse translation of the wedges, allowing extremely high delay accuracy.

The schematic of our FT spectrometer is shown in Fig. 2. The TWINS device, using $\alpha$-barium borate ( $\alpha$-BBO) as the birefringent material, is arranged according to the scheme of Fig. 1(c). Block A has a thickness of $1.6 \mathrm{~mm}$. The wedges have an apex angle of $\alpha=7^{\circ}$ and a length of $25 \mathrm{~mm}$. The moving wedge is mounted on a translation stage (LMS-60 Linear Motor Stage, Physik Instrumente) giving a maximum speed of 500 $\mathrm{mm} / \mathrm{s}$ and uni-directional position repeatability down to $0.1 \mu \mathrm{m}$. We typically scan the wedge at $10-20 \mathrm{~mm} / \mathrm{s}$ constant speed, so that spectra are acquired within $\sim 1 \mathrm{~s}$ measurement time, and then perform further averaging until the desired accuracy is achieved. Considering the apex
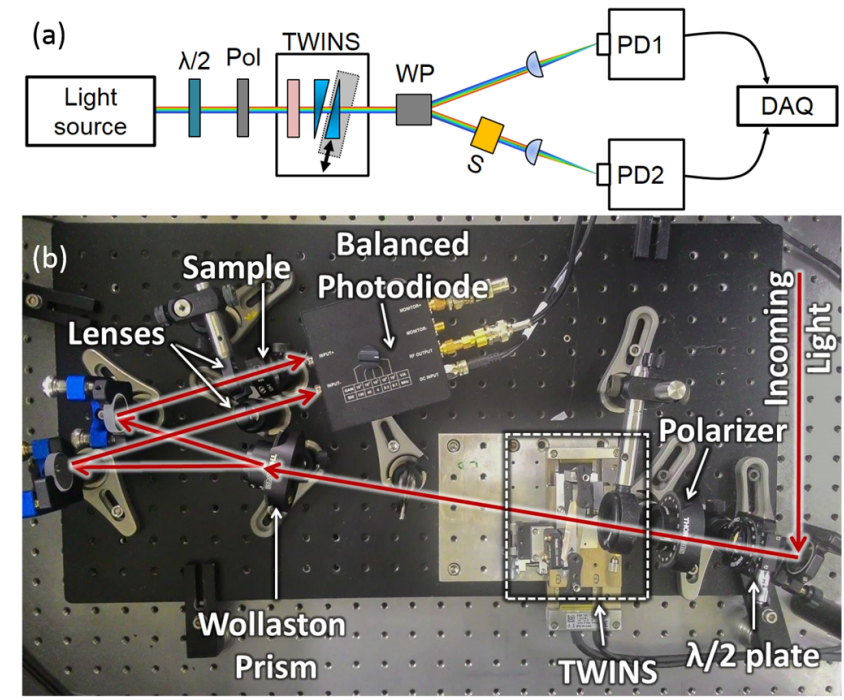

Fig. 2. (a) Schematic setup of the FT spectrometer using TWINS; $\lambda / 2$ : half-wave plate; Pol: polarizer at $45^{\circ}$ with respect to the optical axes of TWINS; WP: Wollaston prism; S: sample; PD1 and PD2: photodiodes; DAQ: data acquisition card. (b) Picture of the setup. angles of the wedges and the birefringence of $\alpha$-BBO, this corresponds to a delay accuracy of $\sim 5$ attoseconds (or $\sim \lambda / 300$ ) at $500 \mathrm{~nm}$. A pinhole placed before block $A$ sets the beam spot size to a diameter of $1.5-2 \mathrm{~mm}$ on blocks $A$ and $B$, which allows a lateral excursion of the moving wedge greater than $20 \mathrm{~mm}$. A half-wave plate and a polarizer placed before the TWINS ensure that the polarization of the input beam is at $45^{\circ}$ with respect to the optical axes of blocks $A$ and B. In a standard TWINS setup, a second polarizer, aligned at $45^{\circ}$ with respect to the orthogonal polarization directions, projects the two delayed replicas onto a common polarization direction, allowing one to observe their interference (see polarizer "P" in Fig. 1(b)). Here we use a WP instead, with optical axes aligned at $45^{\circ}$ with respect to those of the TWINS. In this way, the two spatially separated output beams are equivalent to the projection of the light field along the $+45^{\circ}$ and $-45^{\circ}$ polarization states, respectively, so that no power is lost. The two beams are then measured using the two photodiodes of a balanced silicon detector (PDB450A, Thorlabs, Inc.): its linear signal outputs provide two interferograms phase shifted by $\pi$, while its balanced signal output delivers an interferogram with double amplitude and zero offset [33]. Most importantly, the simultaneous availability of two interferograms allows one to readily record absorption spectra of samples in a single measurement, as explained in the following.

\section{RESULTS}

We first verified the achievable spectral resolution of the spectrometer measuring the line of a He-Ne laser emitting at $633 \mathrm{~nm}$. Figure 3(a) shows a close-up of the two $\pi$-phase-shifted (due to energy conservation) interferograms near delay zero, as recorded by the two photodiodes. The corresponding spectrum obtained by FT of one of the two traces (see Fig. 3(b)) has a full width at half maximum of $2.5 \mathrm{~nm}$. The spectral resolution is inversely proportional to the travel range of the wedges: in this case, the total scan range was $22.5 \mathrm{~mm}$, only limited by the lateral size of the available wedges, corresponding to a total delay between the replicas of $\sim 1.19 \mathrm{ps}$ at $633 \mathrm{~nm}$. Higher resolution than $2.5 \mathrm{~nm}$ could be readily achievable by using either a material with larger birefringence or by changing the design of the wedges (bigger size, larger apex angle). By properly aligning the wedges according to the procedure described in [32], we can achieve a very high contrast in the interference fringes, up to $10^{3}$, as shown in Fig. 3(c). We experimentally found that, once the TWINS have been aligned for the optimal fringe
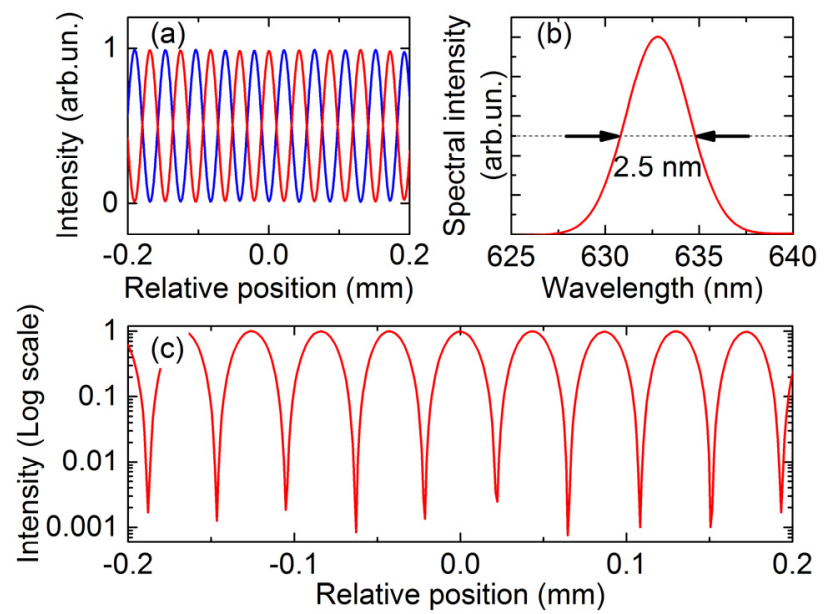

Fig. 3. (a) Zoom of the interferogram traces recorded from the He-Ne laser in a $22.5 \mathrm{~mm}$-long scan from the two photodiodes. (b) Retrieved spectrum. (c) Interferogram trace in logarithmic scale, to highlight the achieved fringe contrast. 
contrast with the He-Ne laser, they provide the same performances for any other beam sent through the same alignment irises. We note that a correct alignment of the wedges is fundamental also for a proper phasing of the interferograms, which would otherwise present residual asymmetries, thus generating an imaginary component of the FT signal.

To demonstrate the capabilities of our FT spectrometer, we first recorded the spectrum of a broadband white-light beam covering more than one octave of bandwidth. As a light source, one could use a simple incoherent lamp, but we preferred to utilize a coherent white-light supercontinuum because it provides higher brightness and lower divergence. We employed a high-power supercontinuum fiber laser system (SuperK Extreme EXW-12 from NKT Photonics), producing a single-mode ultra-broadband spectrum in the 500-2300 nm wavelength range. We selected a portion in the visible and near-IR covering the 500-1000 $\mathrm{nm}$ wavelength range with a suitable shortwave-pass filter, to match the detection range of our silicon photodiode. The recorded interferogram and the corresponding spectrum are reported in Fig. 4(a-b) as blue solid lines.

The spectrum shown is the average out of 300 consecutive acquisitions each performed in just 1-second measurement time. The cyan area in Fig. 4(b) corresponds to the tolerance range within one standard deviation for the measurements with 1 second measurement time, indicating that high accuracy is achievable in a very short amount of time. We then verified the performances of our FT spectrometer in recording transmission spectra of samples. Now the advantage in using a WP for recording absorption spectra is clear: having two identical (except for the $\pi$ phase shift) beams available, one can place the sample to be characterized in one of the two arms (see Fig. 2) and simultaneously measure the light transmitted by the sample. In this way, it is possible to normalize the light transmitted by the sample by the impinging light, thus obtaining the transmission spectrum of the sample with high accuracy because any fluctuation of the spectral intensity of the light is compensated. We chose a BG36 Schott Glass colored bandpass filter (FGB67, Thorlabs, Inc.) presenting a rich
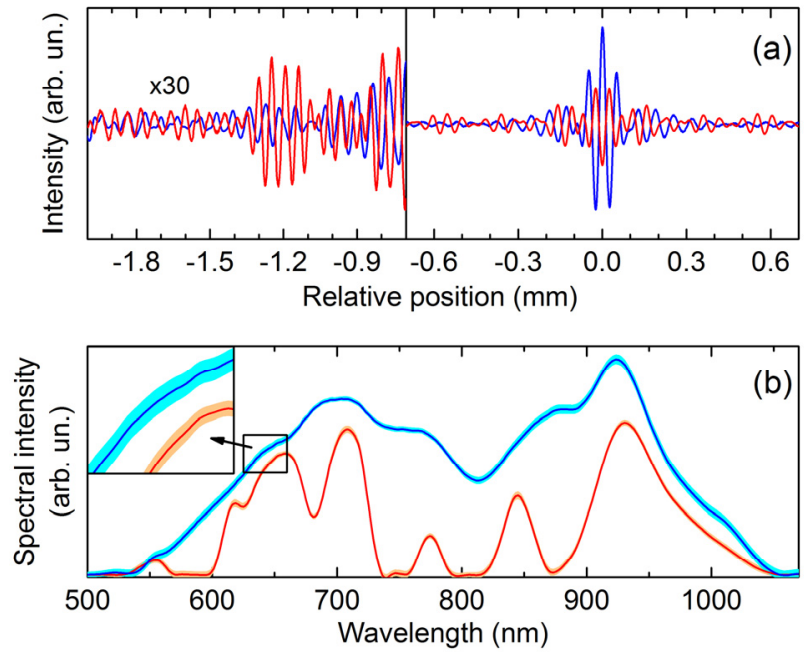

Fig. 4. (a) Interferograms of the white light recorded by the photodiode PD1 (without sample, blue curve) and PD2 (with sample, red curve), shown after subtracting their continuous-wave DC components. A close up (vertical scale multiplied by 30 ) of the interferograms at negative delays is also provided to highlight the fringe pattern that persists also far from time zero. (b) Corresponding spectra, with tolerance ranges (as defined in the text) evaluated over 1 second measurement (cyan and orange areas). A close-up of the spectra is provided to appreciate the thickness of the tolerance areas. spectral structure in the visible and near-infrared wavelength range. Light power at the sample was adjusted to a maximum value of $500 \mu \mathrm{W}$. We limited the scan range to $14 \mathrm{~mm}$, as no features sharper than $5 \mathrm{~nm}$ are present in the spectrum. The recorded interferogram and the corresponding spectrum of the white light transmitted by the filter are shown in Fig. 4(a-b) as red solid lines.

We note that, as expected, this interferogram, with respect to the previous one, presents a more pronounced fringe pattern at longer delays (see the close-up at negative delays in Fig. 4(a)), due to the corresponding richer spectral structure (see Fig. 4(b)). The resulting transmission spectrum of the color filter, calculated as the ratio of the two spectra simultaneously measured by the two photodiodes, is reported in Fig. 5. The orange solid line is the average transmission spectrum out of 300 consecutive measurements performed by moving the wedge at a constant speed of $14 \mathrm{~mm} / \mathrm{s}$, corresponding to 1 second measurement time for each measurement. The gray area is the corresponding tolerance range within one standard deviation for the measurements with 1 second measurement time. This is in the 1-3\% range in the $600-950 \mathrm{~nm}$ spectral region, while it increases considerably below $600 \mathrm{~nm}$ due to relatively low light intensity in this wavelength range. This means that in a few minutes total averaging time we can achieve very high accuracy in the transmission measurements of the order of $0.1 \%$. As a reference, the sample transmission spectrum recorded by a conventional spectrophotometer (model V-570, Jasco Inc.) is also reported in Fig. 5 as a black dashed curve: the quantitative agreement is excellent. We note that, since the TWINS operate in a partially rotating frame [30], a minimum of two known optical frequencies must be employed to calibrate the wavelength axis. We therefore used this color filter to calibrate our FT spectrometer, as done in [34]. For small bandwidths (such as the $520-680 \mathrm{~nm}$ wavelength range used in [32]) a linear interpolation of the calibration curve is sufficient (see Fig. 5(b) in [32]), because the birefringence $\Delta n=n_{0}-n_{e}$ can be considered constant. In our case a polynomial curve is required instead, because in $\alpha$-BBO the birefringence varies from $\Delta \mathrm{n} \sim 0.120$ at the red edge of our spectral window $(1000 \mathrm{~nm})$ to $\Delta \mathrm{n} \sim 0.125$ at the blue edge (at $500 \mathrm{~nm}$ ). The group velocity mismatch $G V M=1 / v_{\text {go }}-$

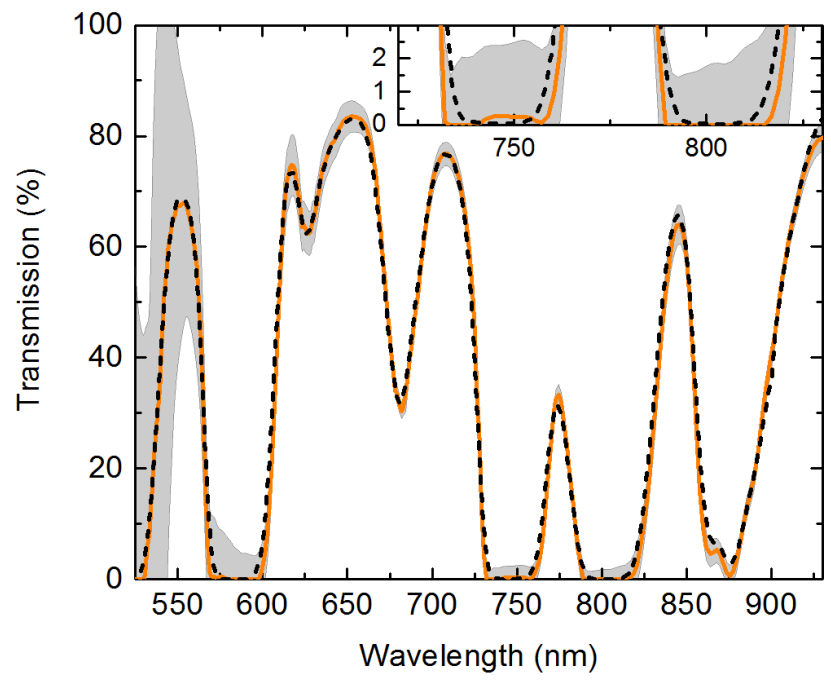

Fig. 5. Transmission spectrum of the sample (orange solid line) averaged over $300 \mathrm{~s}$ and tolerance range (as defined in the text) evaluated over 1 second measurement (gray area), compared with the transmission spectrum measured by a standard spectrophotometer (black dashed line). Inset: magnification of the low transmission values in the $720-830 \mathrm{~nm}$ spectral range. 
$1 / v_{g e}$ between the ordinary and extraordinary components accordingly varies from $\sim 407 \mathrm{fs} / \mathrm{mm}$ at $1000 \mathrm{~nm}$ to $\sim 468 \mathrm{fs} / \mathrm{mm}$ at 500 $\mathrm{nm}$. Following eq. (5), the total delay introduced by the moving wedge (for a lateral displacement of $\mathrm{L}=22.5 \mathrm{~mm}$ ) thus corresponds to $\tau=$ $G V M \cdot L \cdot 2 \tan \frac{\alpha}{2}$, where $\alpha=7^{\circ}$ is its apex angle. This turns out to be equal to $\tau \sim 1.12$ ps at $1000 \mathrm{~nm}$ and $\tau \sim 1.29$ ps at $500 \mathrm{~nm}$, with a nonlinear dependence on wavelength. We therefore interpolated eight known wavelengths of the spectrum of the filter by a third-order polynomial, which provided a very accurate calibration of the wavelength axis. We also mention that, to obtain transmission spectra of samples with high accuracy, an intensity pre-calibration of the setup without any sample must be performed, to correct for any spectral unbalancing of the two beams separated by the WP and for the different responsivities of the two photodiodes.

\section{CONCLUSIONS AND OUTLOOK}

To conclude, we have developed and experimentally validated an FT spectrometer in the visible range, based on the TWINS passive birefringent interferometer. We measured spectra of white-light supercontinua over more than one octave in a short amount of time and we retrieved the transmission spectrum of a reference color filter with high accuracy. Due to the exceptional intrinsic phase stability of TWINS, our spectrometer does not require any active feedback or path-length tracking. With respect to other techniques based on dispersive elements or spatial-domain FT, our spectrometer shows advantages and drawbacks, so that depending on the intensity and stability of the available illumination source the choice of the most suited instrument could vary. Using suitable detectors, our concept can work over the entire transparency range of $\alpha-\mathrm{BBO}$, from $190 \mathrm{~nm}$ to $3 \mu \mathrm{m}$. Using different materials with increased long-wavelength transparency, such as lithium niobate up to $5 \mu \mathrm{m}$ [31] and $\mathrm{Hg}_{2} \mathrm{Cl}_{2}$ (calomel) up to $20 \mu \mathrm{m}$ [36], it can be extended to the mid-IR, covering the crucial fingerprint region of molecular vibrations. With respect to standard FTIR spectrometers in the mid-IR, our approach would not require a MI and position tracking with an accessory laser and would thus be more compact and stable. Finally, besides its already demonstrated uses in nonlinear spectroscopy [31-33, 35], TWINS can be used to replace MIs in a number of applications requiring high delay stability, such as stepscan FTIR spectroscopy, which is a powerful tool to study photoinduced reactions occurring on the nanosecond to microsecond timescale [37].

Funding. European Research Council (ERC) (291198, 648615, 665635); Schweizerischer Nationalfonds zur Förderung der Wissenschaftlichen Forschung (SNF) (PBZHP2_143444).

Acknowledgment. J. R. thanks the Swiss National Science Foundation for financial support (Fellowship PBZHP2_143444).

\section{References}

1. S. P. Davis, M. C. Abrams, and J. W. Brault, "Fourier Transform Spectrometry" (Academic Press, 2001).

2. R. J. Bell, "Introductory Fourier Transform Spectroscopy" (Academic Press, 1972).

3. P. Connes, "Astronomical Fourier Spectroscopy", Ann. Rev. Astron. Astr. 8, 209-230 (1970).

4. R. R. Ernst and W. A. Anderson, "Application of Fourier Transform Spectroscopy to Magnetic Resonance", Rev. Sci. Instrum. 37, 93-102 (1966).

5. A. G. Marshall, C. L. Hendrickson, and G. S. Jackson, "Fourier Transform ion cyclotron resonance mass spectrometry: a primer", Mass Spectrom. Rev. 17, 1-35 (1998).
6. Z. Movasaghi, S. Rehman, and I. U. Rehman, "Fourier Transform Infrared (FTIR) spectroscopy of biological tissues", Appl. Spectrosc. Rev. 43, 134179 (2008).

7. D. Helm, H. Labischinski, G. Schallehn, and D. Naumann, "Classification and identification of bacteria by Fourier Transform Infrared Spectroscopy", Microbiology 137, 69-79 (1991).

8. C. D. Boone, R. Nassar, K. A. Walker, Y. Rochon, S. D. McLeod, C. P. Rinsland, and P. F. Bernath, "Retrievals for the atmospheric chemistry experiment Fourier-transform spectrometer", Appl. Opt. 44, 7218-7231 (2005).

9. P. Y. Han, M. Tani, M. Usami, S. Kono, R. Kersting, and X.-C. Zhang, "A direct comparison between terahertz time-domain spectroscopy and farinfrared Fourier transform spectroscopy", J. Appl. Phys. 89, 2357-2359 (2001).

10. P. B. Fellgett, "On the ultimate sensitivity and practical performance of radiation detectors", J. Opt. Soc. Am. 39, 970-976 (1949).

11. J. F. de Boer, B. Cense, B. H. Park, M. C. Pierce, G. J. Tearney, and B. E. Bouma, "Improved signal-to-noise ratio in spectral-domain compared with time-domain optical coherence tomography", Opt. Lett. 28, 20672069 (2003).

12. P. Jacquinot, "New developments in interference spectroscopy", Rep. Prog. Phys. 23, 267-312 (1960).

13. P. G. Lucey, K. A. Horton, and T. Williams, "Performance of a long-wave infrared hyperspectral imager using a Sagnac interferometer and an uncooled microbolometer array", Appl. Opt. 47, F107-F113 (2008).

14. M. J. Persky, "A review of space infrared Fourier transform spectrometers for remote sensing", Rev. Sci. Instrum. 66, 47634797 (1995).

15. B. C. Smith, "Fundamentals of Fourier Transform Infrared Spectroscopy" (CRC Press, 2011).

16. R. P. Cageao, J. F. Blavier, J. P. McGuire, Y. B. Jiang, V. Nemtchinov, F. P. Mills, and S. P. Sander, "High-resolution Fourier-transform ultraviolet-visible spectrometer for the measurement of atmospheric trace species: application to $\mathrm{OH}^{\prime \prime}$, Appl. Opt. 40, 20242030 (2001).

17. R. K. Chan, P. K. Lim, X. Wang, and M. H. Chan, "Fourier transform ultraviolet-visible spectrometer based on a beam-folding technique", Opt. Lett. 31, 903-905 (2006).

18. X. Wang, R. K. Y. Chan, and A. S. K. Cheng, "Near UV-near IR Fourier transform spectrometer using the beam-folding position-tracking method based on retroreflectors", Rev. Sci. Instrum. 79, 123108 (2008).

19. J. Mandon, G. Guelachvili, and N. Picqué, "Fourier transform spectroscopy with a laser frequency comb", Nature Photon. 3, 99 102 (2009).

20. M. J. Padgett and A. R. Harvey, "A static Fourier-transform spectrometer based on Wollaston prisms", Rev. Sci. Instrum. 66, 2807-2811 (1995).

21. X. Lin, F. Zhou, H. Li, and H. Zhao, "Static Fourier-transform spectrometer based on Wollaston prism", Int. J. Light Electron Opt. 125, 3482-3484 (2014).

22. N. Ebizuka, M. Wakaki, Y. Kobayashi, and S. Sato, "Development of a multichannel Fourier transform spectrometer", Appl. Opt. 34, 7899-7906 (1995).

23. L. Mertz, "Astronomical photoelectric spectrometer", Astron. J. 71, 749-751 (1966)

24. M. F. A'Hearn, F. J. Ahern, and D. M. Zipoy, "Polarization Fourier spectrometer for astronomy", Appl. Opt. 13, 1147-1157 (1974).

25. A. R. Harvey and D. W. Fletcher-Holmes, "Birefringent Fouriertransform imaging spectrometer", Opt. Express 12, 5368-5374 (2004).

26. M. W. Kudenov and E. L. Dereniak, “Compact real-time birefringent imaging spectrometer", Opt. Express 20, 17973-17986 (2012).

27. J. Li, J. Zhu, C. Qi, C. Zheng, B. Gao, Y. Zhang, and X. Hou, “Compact static imaging spectrometer combining spectral zooming capability 
with a birefringent interferometer", Opt. Express 21, 10182-10187 (2013).

28. G. Zhan, K. Oka, T. Ishigaki, and N. Baba, "Birefringent imaging spectrometer", Appl. Opt. 41, 734-738 (2002).

29. J. Chandezon, J.-M. Rampnoux, S. Dilhaire, B. Audoin, and Y. Guillet, "In-line femtosecond common-path interferometer in reflection mode", Opt. Express 23, 27011-27019 (2015).

30. D. Brida, C. Manzoni, and G. Cerullo, "Phase-locked pulses for twodimensional spectroscopy by a birefringent delay line", Opt. Lett. 37, 3027-3029 (2012).

31. J. Réhault, M. Maiuri, C. Manzoni, D. Brida, J. Helbing, and G. Cerullo, "2D IR spectroscopy with phase-locked pulse pairs from a birefringent delay line", Opt. Express 22, 9063-9072 (2014).

32. J. Réhault, M. Maiuri, A. Oriana, and G. Cerullo, "Two-dimensional electronic spectroscopy with birefringent wedges", Rev. Sci. Instrum. 85, 123107 (2014).

33. J. Réhault, F. Crisafi, V. Kumar, G. Ciardi, M. Marangoni, G. Cerullo, and D. Polli, "Broadband stimulated Raman scattering with Fouriertransform detection", Opt. Express 23, 25235-25246 (2015).

34. U. Megerle, I. Pugliesi, C. Schriever, C.F. Sailer, and E. Riedle, "Sub$50 \mathrm{fs}$ broadband absorption spectroscopy with tunable excitation: putting the analysis of ultrafast molecular dynamics on solid ground", Appl. Phys. B 96, 215-231 (2009).

35. F. Preda, V. Kumar, F. Crisafi, D. G. Figueroa del Valle, G. Cerullo and Dario Polli, "Broadband pump-probe spectroscopy at $20-\mathrm{MHz}$ modulation frequency", Opt. Lett. In press (2016)

36. R. Maksimenka, P. Nuernberger, K. F. Lee, A. Bonvalet, J. Milkiewicz, C. Barta, M. Klima, T. Oksenhendler, P. Tournois, D. Kaplan, and M. Joffre, "Direct mid-infrared femtosecond pulse shaping with a calomel acousto-optic programmable dispersive filter", Opt. Lett. 35, 3565-3567 (2010).

37. K. Ataka, T. Kottke, and J. Heberle, "Thinner, Smaller, Faster: IR Techniques To Probe the Functionality of Biological and Biomimetic Systems", Angew. Chem. Int. Ed. 49, 5416-5424 (2010). 